Educación Física y Ciencia, vol. 23, n³, e192, julio-septiembre 2021. ISSN 2314-2561

Universidad Nacional de La Plata.

Facultad de Humanidades y Ciencias de la Educación.

Departamento de Educación Física

\title{
Combinação do Mat Pilates e Tai Chi Chuan na melhoria da qualidade do sono em idosos
}

\author{
Combination of Mat Pilates and Tai Chi Chuan for improving sleep quality in the elderly \\ Combinación del Mat Pilates y Tai Chi Chuan en la mejoría de la calidad del sueño en ancianos
}

\author{
Sara Teresinha Corazza \\ Centro de Educação Física e Desportos (CEFD) da \\ Universidade Federal de Santa Maria (UFSM), Brasil \\ saratcorazza@gmail.com \\ (DD https://orcid.org/0000-0002-2684-2412
}

\author{
Damiana Lima Costa \\ Centro de Ciências da Saúde e do Esporte da \\ Universidade do Estado de Santa Catarina (UDESC), Brasil \\ damilimacosta@hotmail.com
}

(iD https://orcid.org/0000-0001-8276-073X

\author{
Silvane Vagner Vasconcellos \\ Centro de Educação Física e Desportos (CEFD) da \\ Universidade Federal de Santa Maria (UFSM), Brasil \\ silvanevvasconcellos@hotmail.com
}

(iD https://orcid.org/0000-0003-3291-0431

\author{
Evander Vieira de Oliveira \\ Centro de Educação Física e Desportos (CEFD) da \\ Universidade Federal de Santa Maria (UFSM), Brasil \\ evander-@outlook.com
}

iD https://orcid.org/0000-0003-0027-6376

\section{Resumo:}

Desordens no sono, assim como a insônia e sonolência excessiva diurna, são elementos que afetam a qualidade de vida do idoso, porém já existem fortes indícios de que esses problemas podem ser eliminados ou diminuídos com a prática do exercício físico. Objetivo: Verificar a melhoria na qualidade do sono, em um grupo de idosos, após a intervenção combinada do Mat Pilates (MP) e Tai Chi Chuan (TCC). Metodologia: Esta pesquisa caracteriza-se como quase-experimental com pré e pós teste, com 66 idosos, com média de idade de 66,53 anos, que foram submetidos à 26 sessões da prática combinada do MP e TCC e foram submetidos à aplicação do Questionário de Índice de Qualidade do Sono de Pittsburgh (PSQI) antes e após a intervenção. A análise estatística utilizada foi o teste $t$ para amostras pareadas, através do SPSS, na versão 21.0, com nível de significância de 5\%. Resultados e conclusões: Como resultados obteve-se melhora em todas as 7 categorias do PSQI, ressaltando a eficiência da combinação do MP e TCC para um sono reparador e relaxante, melhorando com isso a qualidade de vida e saúde dos idosos.

Palavras-Chave: Idosos, Qualidade do sono, Mat Pilates, Tai Chi Chuan.

\section{AbstraCt:}

Sleep disorders, as well as insomnia and excessive daytime sleepiness, are elements that affect the quality of life of the elderly, but there is already strong evidence that these problems can be eliminated or reduced with physical exercise practice. Objective: the improvement in sleep quality was confirmed in a group of elderly people after the combined intervention of Mat Pilates (MPS)

\section{Recepción: 10 de junio de 2021 | Aprobación: 13 de junio de 2021 | Publicación: 01 de julio de 2021}

Cita sugerida: Corazza, S. T., Costa, D. L., Vasconcellos, S. V. y Vieira de Oliveira, E. (2021). Combinação do Mat Pilates e Tai Chi Chuan na melhoria da qualidade do sono em idosos. Educación Física y Ciencia, 23(3), e192. https://doi.org/10.24215/23142561e192 
and Tai Chi Chuan (TCC). Method: this was a cross-sectional, interventional study with 66 elderly people, with an average age of 66.53 years, who underwent twenty-six sessions of combined practice of MPS and CBT and were submitted to the application of the Quality Index Questionnaire of Pittsburgh Sleep (PSQI) before and after the intervention. The statistical analysis used was the $t$ test for paired samples, using SPSS, in its version 21.0, with a significance level of 5\%. Results and conclusion: as a result, improvement was obtained in all seven categories of the PSQI, highlighting the efficiency of the combination of MP and TCC for restful and relaxing sleep, thereby improving the quality of life and health of elderly people.

KEYWORDS: Elderly, Quality of life, Mat Pilates, Tai Chi Chuan.

\section{RESUMEN:}

Disturbios del sueño, así como el insomnio y somnolencia excesiva, son elementos que afectan la calidad de vida del anciano, pero ya existen fuertes indicios de que estos problemas pueden ser eliminados o disminuidos con la práctica del ejercicio físico. Objetivo: Verificar la mejoría de la calidad del sueño, en un grupo de ancianos, después de la intervención combinada del Mat Pilates (MP) y Tai Chi Chuan (TCC). Metodología: Se trata de una investigación cuasi-experimental con pre y post test, con 66 ancianos, con media de edad de 66,53 años, que fueron sometidos a 26 sesiones de la practica combinada del MP y TCC y fueron sometidos a la aplicación del Cuestionario del Índice de Calidad del Sueño de Pittsburgh (PSQI) antes y después de la intervención. El análisis estadístico utilizado fue el test $\mathrm{t}$ para muestras pareadas, a través del SPSS, en la versión 21.0, con nivel de significancia de $5 \%$. Resultados y conclusiones: Como resultados se obtuvo mejoría en todas las 7 categorías del PSQI, principalmente la eficacia de la combinación del MP y TCC para un sueño reparador y relajante, mejorando con esto la calidad de vida y salud de los ancianos.

Palabras Clave: Ancianos, Calidad del sueño, Mat Pilates, Tai Chi Chuan.

\section{INTRODUÇÃO}

O envelhecimento representa um conjunto de consequências ou de efeitos da passagem do tempo. Ele pode estar associado à muitos fatores, como moleculares sistêmicos, comportamentais, cognitivos e sociais (Ramos, 2003). Um dos elementos comportamentais que exerce uma influência impactante na qualidade de vida durante o processo de envelhecimento é o sono (Unruh et al., 2008).

O sono possui um papel muito importante na homeostasia, cujo desequilíbrio favorece o aparecimento de transtornos ou confusões mentais, diminuição da competência imunológica, prejuízo no desempenho físico e dificuldades adaptativas, causando aumento da vulnerabilidade do organismo idoso e provocando riscos à sua vida (Quinhones e Gomes, 2011). Pode-se inclusive, com o envelhecimento normal, segundo Bertolli Filho (2004) ter ocorrências de perdas gerais do relógio circadiano, ocasionando aumento da fragmentação do sono, necessidade de aumentar os cochilos durante o dia, alteraçóes nas fases de deitar e levantar, maior fadiga diurna e dessincronização das atividades sociais. Existem evidências crescentes apontando para a redução na duração das horas de sono concomitantemente com o aumento da prevalência de desordens do sono, em populações idosas (Jurado et al., 2020).

À vista disso, a prevalência de desordens do sono é um problema presente e relevante em nossa sociedade, visto que tem se tornado uma queixa comum entre os idosos, uma vez que durante o processo de envelhecimento, causas como incontinência urinária, apneia do sono, dor ou desconforto físico e emocional, parassonia, distúrbios no ritmo circadiano, doenças articulares, necessidade de tomar medicamentos durante a madrugada, fatores ambientais entre outros podem provocar alterações no padrão de sono (Silva et al., 2017; Moreno et al., 2018). Sendo assim, torna-se importante a busca por estratégias que proporcionem uma melhora na qualidade do sono dos idosos e ajudem a reduzir a prevalência destas alterações nessa população.

Pesquisas recentes têm indicado a importância do exercício físico na promoção da saúde e qualidade de vida de idosos. Sua prática regular está associada diretamente ao aumento da longevidade (Haskell et al., 2007; Teculescu et al., 2010). Entretanto existem algumas incertezas em relação à qual é o exercício mais benéfico (Martins et al., 2001). Uma análise bidirecional tem apontado para uma correlação entre atividade física e o sono, apontando efeitos positivos na diminuição do uso de medicação e na latência do sono (Christopher, 2014; Kovacevic et al., 2017). Além disso a American Sleep Disorders Association, traz a importância do 
exercício físico como uma intervenção de qualidade, não-farmacológica, para melhorar o padrão do sono, no entanto ressalta a ausência de uma prescrição específica com essa finalidade.

$\mathrm{Na}$ busca por exercícios físicos adequados aos idosos e que trouxessem benefícios à qualidade do sono, nos deparamos com duas práticas bem impactantes e de fácil execução, o Método Pilates (Röpke, 2017; Sousa $e t$ al., 2019; Kneip et al., 2018) e a prática do Tai Chi Chuan (Kit, 2016). Esse primeiro tem sido visto como um importante conjunto de exercícios promotor da saúde e qualidade de vida à população idosa, caracterizandose por trabalhar com a respiração, concentração, controle corporal, precisão e fluidez, integrando corpo e mente (McNeill, 2011). O Tai Chi Chuan (TCC) já está sendo bastante explorado com a população idosa, tratando-se de uma prática milenar chinesa, de baixo impacto, com movimentos lentos, bem alongados, suaves, explorando as técnicas respiratórias (Kit, 2016) e também indicativo para um sono reparador (Du et al., 2015; Hosseini et al., 2011). Essa prática inclui em seu conjunto de exercícios a preocupação com o corpo e a mente, regulação do fluxo de energia, aumento da longevidade, melhoria da consciência corporal, atenção mental, relaxamento e profunda respiração diafragmática.

Portanto, por termos evidências de que tanto o Mat Pilates (MP) quanto o Tai Chi Chuan (TCC) podem trazer inúmeros benefícios aos idosos, propõem-se como meta desse estudo a verificação na qualidade do sono através da combinação desses dois métodos.

\section{Metodologia}

Esta pesquisa caracteriza-se como um estudo quase-experimental com pré e pós teste (Sousa et al., 2007). A amostra foi composta por 66 idosos do sexo feminino e masculino, com idade entre 62 e 84 anos, com média de idade de 66,53 anos e desvio padrão de 5,12. Esses foram selecionados de forma intencional, sendo os indivíduos convidados a participar da pesquisa através de mídias sociais, divulgação em jornais e grupos de convivência numa cidade da região sul do Brasil. A seleção baseou-se nos seguintes critérios de inclusão: ter idade igual ou superior a 60 anos, não ser praticante de exercício físico regular, apresentar atestado médico declarando condições saudáveis para prática do MP e do TCC, ter autonomia para deslocar-se e executar as tarefas físicas e motoras e disponibilidade para frequentar as aulas do MPS e TCC duas vezes por semana. Adotou-se como critério de exclusão a presença de qualquer restrição para a prática das atividades propostas e ter frequência às aulas inferior a $75 \%$.

A intervenção teve 26 aulas combinando exercícios Mat Pilates (MP) e Tai Chi Chuan (TCC). As testagens aconteceram na semana anterior ao início das aulas, caracterizando-se como pré-teste e na semana posterior à última aula, caracterizando-se como pós-testes, sendo todas realizadas pelos mesmos avaliadores, os quais foram previamente treinados. Os dados foram coletados, no laboratório de Aprendizagem Motora (sala 1021), do Centro de Educação Física (CEFD) da Universidade Federal de Santa Maria (UFSM).

Para a avaliação do sono foi utilizado o Questionário Índice de Qualidade do Sono de Pittsburgh (PSQI) que avalia a qualidade e perturbações o sono. Esse instrumento é composto por 19 questões em auto relato e cinco questões direcionadas ao cônjuge ou acompanhante de quarto. As últimas 5 questões não foram utilizadas nessa análise, pois elas são de competência somente à prática clínica. As 19 questões foram categorizadas em 7 componentes, graduados em escores de zero (nenhuma dificuldade) a três (dificuldade grave). Os 7 componentes do PSQI são qualidade subjetiva do sono (QS), latência do sono (LS), duração do sono (DS), eficiência dos sono (ES), alterações do sono (AS), uso de medicamentos para indução do sono (MS) e disfunção diurna (DD) provocada por noite mal dormidas. A análise total dessas categorias pode resultar num índice que varia de 0 a 21 pontos indicando que quanto maior o número pior é a qualidade do sono (Buysse et al., 1989).

As aulas do Mat Pilates (MP) e Tai Chi Chuan (TCC), caracterizando a intervenção, aconteceram de setembro a dezembro de 2019, no Complexo Didático e Artístico - Dança (CDA) do Centro de Educação Física e Desporto (CEFD) da Universidade Federal de Santa Maria (UFSM). Na combinação dos dois 
métodos houve a presença de duas profissionais da Educação Física e uma profissional da Fisioterapia que ministraram as aulas. Quatro acadêmicos do Curso de Bacharelado em Educação Física auxiliaram durante a intervenção, ressaltando cuidados posturais e orientando a correta e completa execução dos exercícios.

As atividades ocorreram duas vezes por semana (60 minutos) utilizando $20 \mathrm{~min}$ de exercícios de Tai Chi Chuan e 40 min de Mat Pilates. Os exercícios utilizados nas aulas foram os do MP, que consiste em exercícios realizados normalmente em colchonetes, exigindo consciência corporal, força, alongamento e equilíbrio. Os exercícios foram realizados em decúbito dorsal, ventral, quatro apoios, ajoelhados e sentados. Nas primeiras aulas não houve utilização de nenhum acessório, posteriormente, foram introduzidas faixas elásticas de tensão forte, média e leve e bolas suíças. Os exercícios contaram com a utilização do peso do próprio corpo. A aplicação ocorreu de modo progressivo e com a evolução dos exercícios de menor dificuldade para os de maior, respeitando os níveis de aptidão física de cada um, sendo que, as primeiras aulas foram de adaptação, com ênfase na respiração e ativação do centro de força ou power house. Nas aulas seguintes, foram adicionados mais exercícios.

O Tai Chi Chuan foi aplicado utilizando-se de movimentos alongados, lentos e rítmicos com o objetivo de desenvolver o fluxo interno de energia e buscando enfatizar a rotação do tronco, a coordenação e o estreitamento gradual da postura dos membros inferiores. Os exercícios priorizaram a postura correta, treinamento de força, coordenação de braços/pernas e equilíbrio estático/dinâmico. O método foi desenvolvido com a segmentação dos movimentos, partindo de situações mais simplificadas para a construção de rotinas com envolvimento de todo corpo e com deslocamentos no espaço. O método de base na seleção dos exercícios foi o Chi Kung (Kit, 2016).

Durante toda a intervenção, os idosos foram estimulados por meio de informações, tanto verbais quanto visuais, a colocar em prática os princípios de respiração, controle, concentração, precisão, fluidez e centralização, e assim vivenciar holisticamente corpo e mente. Inclusive, os mesmos também foram incentivados a se conscientizar quanto a postura, força, resistência, flexibilidade e equilíbrio, contribuindo assim, para uma melhor estabilidade da saúde, bem como da aptidão física.

O projeto de pesquisa foi submetido e aprovado pelo Comitê de Ética em Pesquisa da Universidade Federal de Santa Maria sob CAAE número: 45429015.4.0000.5346. Todas as participantes do estudo leram e assinaram o Termo de Consentimento Livre e Esclarecido (TCLE).

Para a análise dos resultados foi primeiramente realizada a análise da média e desvio padrão de todas variáveis, após, os dados foram analisados através do teste de Shapiro Wilk para a identificação da normalidade, que mostrou que estes podem ser considerados com distribuição normal. Após, as variáveis foram comparadas antes e após a intervenção através do teste t para amostras pareadas. Utilizou-se o programa SPSS, na versão 21.0, com nível de significância de 5\%.

\section{Resultados}

Apresenta-se na tabela 1 os dados sociodemográficos dos 66 indivíduos participantes desse estudo. Nessa, percebeu-se a maior ocorrência de mulheres idosas; quanto ao estado civil, a grande maioria é casada, seguido de viuvez e o ensino médio, seguido do ensino fundamental detém o predomínio quanto ao nível de escolaridade. 
TABELA 1

Dados sociodemográficos dos 66 participantes do estudo

\begin{tabular}{lll}
\hline Variáveis & $\begin{array}{l}\text { Frequência } \\
\text { relattva (\%) }\end{array}$ & $\begin{array}{l}\text { Frequência } \\
\text { absoluta }\end{array}$ \\
\hline Sexo & 83,6 & 56 \\
$\quad$ Feminino & 16,4 & 10 \\
Masculino & & \\
& & \\
Estado civil & 36,8 & 24 \\
$\quad$ Casado(a) & 16,4 & 11 \\
Solteiro(a) & 30,4 & 20 \\
Viúvo(a) & 16,4 & 11 \\
Divorciado(a)/separado(a) & & \\
& & 2 \\
Escolaridade & 3,0 & 24 \\
Analfabeto - sem escolaridade & 36,8 & 14 \\
Ensino Fundamental & 38,8 & \\
Ensino Médio & 21.4 & \\
Ensino Superior & & \\
& &
\end{tabular}

Fonte: elaborado pelos autores

$\mathrm{Na}$ tabela 2 apresenta-se os dados descritivos de média e desvio padrão relativos às 7 categorias do Questionário Índice de Qualidade do Sono de Pittsburg (PSQI). Encontra-se também as diferenças estatisticamente significativas entre as 7 variáveis, após a intervenção das 26 sessões apontando para a eficiência da combinação do MPS e TCC na percepção de melhoria da qualidade do sono. 
TABELA 2

Dados descritivos e comparação entre as categorias do PSQI antes e após a intervenção

\begin{tabular}{lllll}
\hline Variáveis & Pré (Média \pm dp) & Pós (Média \pm dp) & t & p \\
\hline & & & & \\
Qualidade subjetiva do sono (QS) & $0,91 \pm 0,54$ & $0,47 \pm 0,53$ & 6,39 & $0,008^{*}$ \\
Latência do sono (LS) & $1,36 \pm 0,98$ & $0,67 \pm 0,64$ & 7,22 & $0,007^{*}$ \\
Duração do sono (DS) & $0,74 \pm 0,81$ & $0,38 \pm 0,48$ & 4,40 & $0,010^{*}$ \\
Efíciência do sono (ES) & $1,64 \pm 0,88$ & $0,89 \pm 0,64$ & 7,60 & $0,007^{*}$ \\
Alterações do sono (AS) & $2,17 \pm 0,62$ & $1,02 \pm 0,61$ & 10,03 & $0,006^{*}$ \\
Uso de medicamentos (MS) & $1,14 \pm 0,98$ & $0,58 \pm 0,44$ & 3,95 & $0,012^{*}$ \\
Disfunção Noturna (DN) & $0,83 \pm 0,77$ & $0,38 \pm 0,54$ & 4,80 & $0,011^{*}$ \\
Total & $8,76 \pm 3,40$ & $4,42 \pm 2,03$ & 13,28 & $0,005^{*}$ \\
\hline
\end{tabular}

Fonte: elaborado pelos autores

${ }^{*}$ Diferença significativa com $\mathrm{p} \leq 0,05$.

\section{Discussão}

O principal propósito do estudo foi verificar a melhoria na qualidade do sono, em um grupo de idosos, após a intervenção combinada do MP e TCC. Os resultados gerais permitiram constatar que houve melhora em todas categorias do questionário de qualidade do sono, quando comparados antes e após a realização de 26 sessões com exercícios respiratórios, de alongamento, equilíbrio, flexibilidade e fortalecimento muscular, conteúdos fundamentais utilizados na combinação das técnicas.

A recente revisão sistemática Lins-Filho et al. (2019), a qual investigou os efeitos do Pilates na qualidade do sono de adultos e idosos, teve como principal resultado que a prática do Pilates através do método Mat Pilates com duração mínima de 4 semanas, duas a três sessões semanais, com um tempo médio de 120 a 180 minutos de atividade por semana, de 13 a 15 exercícios, de 1 a 2 séries, com repetições de 8 a 12, trabalhando o controle da respiração foram suficientes para melhorar a qualidade do sono de adultos e idosos. Tal achado, vem ao encontro dos resultados obtidos no presente estudo, que se assemelha aos estudos mencionados na revisão, quanto ao uso do método, frequência semanal, duração e média de exercícios utilizados.

Em um outro estudo Garcia-Soidán et al. (2014), analisou os efeitos do Método Pilates na qualidade de vida, latência e quantidade do sono, em sujeitos de idade média (47,6 anos) com prática semanal de 2 horas, em 12 semanas, os resultados sugeriram uma expressiva redução na latência do sono, exatamente como no presente estudo e ainda, aumentaram a qualidade do sono, principalmente nos dias em que realizavam a aula do MP. Corroborando com esses resultados Curi et al. (2017), também encontrou melhora na latência do sono e a diminuição de medicamentos, com intervenção de 16 semanas com o MP, com o uso do PSQI e também com população idosa. Pode-se entender a latência como o tempo necessário para realizar a transição entre deitar na cama e dormir, que em geral é entre 10 e $30 \mathrm{~min}$.

Quanto à diminuição no uso de medicamentos, também resultado positivo do presente estudo, percebeuse seu significado a partir da expressão da $5^{\text {a }}$ categoria do PSQL (uso de medicamentos), quando a média baixou de 1,14 para 0,58 e depoimentos dos próprios idosos ao mencionarem que se esforçaram para quebrar o ciclo de dependência no uso da medicação, pois já estavam sentindo efeitos colaterais diurnos, 
principalmente relacionados à memória. De maneira geral, a interrupção do sono é prejudicial para o funcionamento diurno do indivíduo, pois compromete aspectos cognitivos, como a concentração e memória, além das habilidades psicomotoras (Müller e Guimarães, 2007).

Ao analisar os efeitos do MPS na flexibilidade e qualidade do sono, após 15 sessões, com idosos, os autores obtiveram melhorias, verificadas através das categorias totais na análise do PSQI e também na utilização da Escala de Sonolência de Epworth (Kneip et al., 2018). Utilizaram, portanto, dois instrumentos avaliativos e ambos mostraram em suas evidências o quanto as aulas do MPS foram significativas para evitar sonolências diurnas ou cochilos em situações inoportunas, pois garantiram a qualidade e quantidade do sono noturno.

Contrário aos achados tem-se o estudo que buscou a comparação entre o Método Pilates de Solo e a hidroginástica na qualidade do sono tendo a mesma população e instrumento avaliativo do presente estudo e seus resultados não apontaram diferença entre as práticas nem tampouco a melhora da qualidade do sono após a intervenção (Sousa et al., 2019). Percebeu-se que não foi reportado no estudo mencionado a quantidade de sessões do Método Pilates para servir de comparativo de forma mais objetiva.

Atualmente têm-se nas práticas chinesas, com ênfase na respiração, equilíbrio, alongamento e coordenação, fortes indícios de sua importância para a qualidade de vida e do sono em idosos. Numa revisão sistemática sobre melhoria do sono através das práticas do TCC foram encontrados 11 estudos com a utilização do mesmo questionário dessa pesquisa. Todos resultados ressaltaram a importância do TCC na vida de pessoas saudáveis ou com doenças crônicas, apontando para a diminuição de dores em geral, melhoria do bem estar e qualidade do sono (Raman et al., 2013).

Li et al. (2004) encontraram melhoras consideráveis na qualidade do sono através da aplicação do TCC com aulas em 3 dias das semanas, totalizando 24 semanas, com 118 homens idosos. A prática do TCC foi bem semelhante ao presente estudo com deslocamentos laterais e anteroposteriores, exercícios de coordenação de braços e pernas, equilíbrio dinâmicos e estáticos e estímulo à concentração nas técnicas respiratórias, buscando a harmonia entre o movimento, o corpo e a mente.

$\mathrm{Na}$ aplicação de aulas semanais por seis meses de Tai chi qigong em idosos com declínios cognitivos Chan et al. (2016), utilizou o mesmo questionário para a análise do sono e encontrou-se diferença estatisticamente significativa quando comparado grupo com e sem intervenção. Na pesquisa foi destacada a importância de repetir movimentos simples e com espelhamento de movimentos para a ocorrência de feedbacks visuais. Essas características interventivas são idênticas ao presente estudo utilizando exercícios fáceis e simples auxiliando na aprendizagem e retenção de movimentos e informações.

Encontrou-se um estudo com as duas práticas em evidência o MPS e o Taiji quan, porém com estudantes universitários (Caldwell et al., 2007). No estudo foi realizado uma comparação entre os métodos, obtendo resultado positivo em ambas para a melhoria tanto na qualidade do sono e no estado de humor, ressaltando a importância da realização de práticas respiratórias e de alongamento e que se estas forem aplicadas mais próximas ao turno da noite, o sono tranquilo e reparador será favorecido. Considerando dessa forma a combinação dos métodos Pilates e Tai Chi Chuan, no presente estudo, foi priorizado igualmente a técnica respiratória. Acredita-se nela como importante preditora dos resultados significativos de todos os fatores relacionados ao sono.

A revisão sistemática de Vanderlinden et al. (2020), sugere que programas de exercícios afetam positivamente vários aspectos do sono em idosos, principalmente aqueles de intensidade moderada, com frequência de três vezes semanais e duração de 12 semanas a 6 meses, como por exemplo, o Tai Chi Chuan, citado pelos autores por mostrar maior proporção de efeitos significativos na qualidade do sono dessa população.

Com relação aos mecanismos que promovem os efeitos do TCC na qualidade do sono, alguns pressupostos fazem sentido. Do ponto de vista fisiológico, como forma de exercício aeróbio, o TCC é realizado de forma lenta e suavemente com a respiração diafragmática e relaxamento, resultando em declínio da estimulação simpática e aumento da sensação de bem-estar, o que pode levar a uma melhor qualidade do sono (Li $e t$ 
al., 2004; Irwin et al., 2008; Motivala et al., 2006). Da mesma forma, o método Pilates tem como um dos princípios básicos a respiração diafragmática (Wells et al., 2012), essa condição faz com que a prática do método melhore a inspiração, que é um dos fatores associados a baixa qualidade do sono em adultos com apneia do sono, por exemplo (Semelka et al., 2016). Tem-se, portanto, mais uma vez a respiração, presente na combinação dos métodos como importante elemento para a obtenção dos resultados. Além disso, alguns outros mecanismos, como o aumento no consumo de energia, secreção de endorfina e temperatura corporal, também são benéficos para melhorar a qualidade do sono (Yang et al., 2012).

Por fim, para efeito psicológico, o estudo de Wang et al. (2010) concluiu que TCC pode melhorar o bem-estar dos participantes, incluindo redução do estresse, ansiedade, depressão e distúrbios de humor e aumento da autoestima. Igualmente, Vieira et al. (2013) e Pereira et al. (2013) apontam que o Pilates atua diretamente no controle do estresse e na melhora da qualidade de vida, fatores relacionados com a qualidade do sono. Portanto, o bem-estar psicológico também pode desempenhar um papel importante na melhoria da qualidade do sono.

Indivíduos idosos que possuem má qualidade do sono podem ter aumento de cochilos durante o dia, declínios e implicações cognitivas e diminuição das capacidades físicas e motoras, que são marcadores de comprometimentos da qualidade física e mental e estão diretamente relacionados à baixa qualidade de vida em geral, afetando inclusive os relacionamentos socioafetivos (Martins et al., 2001). Esses argumentos sustentam a importância de um sono reparador e relaxante e esse pode ser auxiliado pela prática de exercícios que venham a contemplar a latência, a eficiência e possíveis disfunções noturnas.

\section{Conclusões}

Conforme resultados obtidos nas 7 categorias do Questionário do Índice de Qualidade do Sono de Pittsburgh (PSQI), considerando a prática de exercícios combinando elementos do MP e TCC, obteve-se melhoria expressiva e significativa após 26 sessões, apontando para a eficácia da diversificação de estratégias unindo exercícios de fortalecimento muscular, flexibilidade, alongamento, atenção mental, consciência corporal, respiração profunda e controlada na qualidade do sono de idosos.

Sugere-se, portanto, com base nos resultados e evidências empíricas a estimulação da prática de exercícios físicos com as variáveis já mencionadas, pois estas se traduzem em elementos fundamentais para a qualidade do sono e consequentemente uma boa qualidade de vida durante o processo de envelhecimento.

\section{REFERÊNCIAS}

Bertolli Filho, C. (2004). Corpo, cultura e memória: depoimentos de universitários. Bauru, SP: Coleções FAAC: série em pesquisas vol. 2.

Buysse, D., Reynolds, C., Monk, Berman, S. R., Kupfer, D. J. (1989). The Pittsburgh Sleep Quality Index: a new instrument for psychiatric practice and research. Psychiatry Res, 28, 193-213. https://doi.org/10.1016/01651781(89)90047-4.

Caldwell, K., Harrison, M., Adams, M., Triplett, T. (2007). Effect of Pilates and taiji quan training on self-efficacy, sleep quality, mood, and physical performance of college students. Journal of Bodywork and Movement Therapies, 155-63. https://doi.org/10.1016/j.jbmt.2007.12.001.

Chan, A., Yu, D., Choi, K., Lee, D., Sit, J., Chan, H. (2016). Tai chi qigong as a means to improve night-time sleep quality among older adults with cognitive impairment: a pilot randomized controlled trial. Clin Interv Aging, 11,1277-86. https://doi.org/10.2147/cia.s111927.

Christopher, E. K. (2014). The Bidirectional Relationship Between Exercise and Sleep: Implications for Exercise Adherence and Sleep Improvement. American Journal of Lifestyle Medicine, 8(6), 375-9. https://doi.org/10.1 $177 / 1559827614544437$. 
Curi, V. S., Vilaça, J., Hass, A., Fernandes, H. M. (2018). Effects of 16-weeks of Pilates on health perception and sleep quality among elderly women. Archives of Gerontology and Geriatrics, (74), 118-22. https://doi.org/10.1016/j .archger.2017.10.012.

Du, S., Dong, J., Zhang, H., Jin, S., Xu, G., Liu, Z. (2015). Taichi exercise for selfrated sleep quality in older people: a systematic review and meta-analysis. Int J Nurs Stud, 52(1), 368-79. https://doi.org/10.1016/j.ijnurstu.201 4.05.009.

García-Soidán, J. L., Giraldez, V. A., Zagalaz, J. C., Lara-Sánchez, A. J. (2014). Does Pilates Exercise Increase Physical Activity, Quality of Life, Latency, and Sleep Quantity in Middle-Aged People? Perceptual and Motor Skills [Internet]. SAGE Publications, 119(3), 838-50. http://dx.doi.org/10.2466/29.25.pms.119c30z9.

Haskell, W. L., Lee, I. M., Pate, R. R., Powell, K. E., Bauman, A. (2007). Physical activity and public health: updated recommendation for adults from the American College of Sports Medicine and the American Heart Association. Medicine \& Science in Sports \& Exercise, 1081-93. https://doi.org/10.1161/circulationaha.107.185649.

Hosseini, H., Esfirizi, M., Marandi, S., Rezaei, A. (2011). The effect of Tai Chi exercise on the sleep quality of the elderly residents in Isfahan, Sadeghieh elderly home. Iran J Nurs Midwifery Res, 16(1), 55-60.

Irwin, M. R., Olmstead, R., Motivala, S. J. (2008). Improving sleep quality in older adults with moderate sleep complaints: A randomized controlled trial of Tai Chi Chih. Sleep, 31(7), 1001-1008. https://doi.org/10.5665 /sleep/31.7.1001.

Jurado-Fasoli, L., De-La-O, A., Molina-Hidalgo, C., Migueles, J. A., Castillo, M., Amaro-Gahete. (2020). Exercise training improves sleep quality: A randomized controlled trial. Eur J Clin Invest, 50:e13202. https://doi.org/1 $0.1111 /$ eci.13202.

Kit, K. W. K. (2016). O livro completo do Tai Chi Chuan - um manualpormenorizado dos seus princípios e prática. 1o ed. São Paulo: Ed. Pensamento.

Kneip, K., Contenças, T., Oliveira, D. (2018). The influence of the solo Pilates method on the sedentary elderly in improving flexibility and quality of sleep. Revista Brasileira de Fisiologia do Exercício, 17(1), 38-46. https://doi .org/10.33233/rbfe.v17i1.2367.

Kovacevic, A., Mavros, Y., Heisz, J. J., Fiatarone Singh, M. A. (2017). The effect of resistance exercise on sleep: a systematic review of randomized controlled trials. Sleep Med Rev, 39, 52-68. https://doi.org/10.1016/j.smrv. 2017.07.002.

Li, F., Fisher, K. J., Harmer, P., Irbe, D., Tearse, R., Weimer, B. S. (2004). Tai Chi and Self-Rated Quality of Sleep and Daytime Sleepiness in Older Adults: A Randomized Controlled Trial. JAGS, 52(6), 892-900. https://doi .org/10.1111/j.1532-5415.2004.52255.x.

Lins-Filho, O. L., Queiroz, G. K. F., Santos, J. F. J., Santos, M. A. M., Oliveira, L. M. F. T., Farah, B. Q. (2019). Efeitos do Pilates na qualidade do sono em adultos e idosos: uma revisa\#o sistema\#tica. Rev Bras Ativ Fis Sau\#de, 24:e0080. https://doi.org/10.12820/rbafs.24e0080.

Martins, P., De Mello, M., Tufik, F. (2001). Exercício e sono. Rev Bras Med Esporte, 7(1), 233-43. https://doi.org/ $10.1590 / \mathrm{s} 1517-86922001000100006$.

Mcneill, W. (2011). Decision making in Pilates. J Bodyw Mov Ther, 15(1), 103-7. https://doi.org/10.1016/j.jbmt.2 010.10 .003 .

Moreno, C. R. C., Santos, J. L. F., Lebrão, M.L., Ulhôa, M.A., Oliveira Duarte, Y.A. (2018). Problemas de sono em idosos estão associados sexo feminino, dor e incontinência urinária. Rev. bras. epidemiol., 21(2):e180018. https ://doi.org/10.1590/1980-549720180018.supl.2.

Motivala, S. J., Sollers, J., Thayer, J., Irwin, M. R. (2006). Tai Chi Chih acutely decreases sympathetic nervous system activity in older adults. Journals of Gerontology Series A, 61(11), 1177-1180. https://doi.org/10.1093/gerona/ 61.11.1177.

Müller, M. R., Guimarães, S. S. (2007). Impacto dos Transtornos do Sono sobre o funcionamento diário e a qualidade de vida. Estudos de Psicologia, 24(4), 519-528. https://doi.org/10.1590/s0103-166x2007000400011.

Pereira, E. C., Liberali, R., Lopes, C. R., Cruz, T. M., Netto, M. I., Viana, H. B., Ribero da Mota, G.. (2013). Aderência de pessoas no Pilates solo. Fisio Brasil, 14(1), 53-60. https://doi.org/10.33233/fb.v14i1.369. 
Quinhones, M. S., Gomes, M. M. (2011). Sono no envelhecimento normal e patológico: aspectos clínicos e fisiopatológicos. Rev Bras Neurol, 47(1), 31-42.

Raman, G., Zhang, Y., Minichiello, V. J., D’ Ambrósio, C. M., Wang, C. (2013). Tai Chi Improves Sleep Quality in Healthy Adults and Patients with Chronic Conditions: A Systematic Review and Meta-analysis. J Sleep Disord Ther, 2(6), 1-6. https://doi.org/10.4172/2167-0277.1000141.

Ramos, L. R. (2003). Fatores determinantes do envelhecimento saudável em idosos residentes em centro urbano: Projeto Epidoso, São Paulo. Cad Saúde Pública, 19(3), 793-797. https://doi.org/10.1590/S0102-311X20030 00300011.

Röpke, L. (2017). Avaliação do efeito de um programa de exercícios do método Pilates na qualidade do sono e na qualidade de vida de pacientes com SAOS, em uso adequado de CPAP. [Tese]. http://hdl.handle.net/11449/ 150067.

Semelka, M., Wilson, J., Floyd, R. (2016). Diagnosis and Treatment of Obstructive Sleep Apnea in Adults. Am Fam Physician, 94(5), 355-60. https://doi.org/10.3109/9781420020885-22.

Silva, K. K. M., Lima, G. A. F., Gonçalves, R.G, Paiva de Menezes, R. M, Figueiredo De Martino, M. M. (2017). Alterações do sono e a interferência na qualidade de vida no envelhecimento. Rev enferm UFPE, 11(1):422-428. https://doi.org/10.5205/reuol.7995-69931-4-SM.1101sup201722.

Sousa, V. D., Driessnack, M., Mendes, I. A. C. (2007). Uma visão geral dos projetos de pesquisa relevantes para a enfermagem: Parte 1: projetos de pesquisa quantitativa. Revista Latino-Americana de Enfermagem, 15 (3), 502 507. https://doi.org/10.1590/S0104-11692007000300022.

Sousa, A. J. F., Santos, J. P., Santos-Júnior, F. F. U. (2019). Comparative sleep analysis in elderly practitioners of Pilates or water aerobics exercises: a cross-sectional study. Revista Brasileira de Fisiologia do Exercício, 18(4),180-185. h ttp://dx.doi.org/10.33233/rbfe.v18i4.3113.

Teculescu, D., Chenuel, B., Benamghar, L., Michaely, J.P., Hannhart, B. (2010). Is a sedentary lifestyle a risk factor for sleep-related respiratory disturbance? Revue de Maladies Respiratoires, 27(7),703-8. https://doi.org/10.10 16/j.rmr.2010.07.003.

Thomas, J. R., Nelson, J. K., Silverman, S. J. (2012). Métodos de pesquisa em atividade física. Porto Alegre: Artmed.

Unruh, M. L., Redline, S., An, M. W., Buysse, D.J., Nieto, F. J., Yeh, J. L., Newman, A.B. (2008). Subjective and objective sleep quality and aging in the sleep heart health study. J Am Geriatr Soc, 56(7), 1218-27. https://do i.org/10.1111/j.1532-5415.2008.01755.x.

Vanderlinden, J., Boen, F., Van Uffelen, J. G. Z. (2020). Effects of physical activity programs on sleep outcomes in older adults: a systematic review. Int J Behav Nutr Phys Act 17(11), 1-15. https://doi.org/10.1186/s12966-02 0-0913-3.

Vieira, F. T., Faria, L. M., Wittmann, J. I., Teixeira, W., Nogueira, L. A. (2013). The influence of Pilates method in quality of life of practitioners. J Bodyw Mov Ther, 17(4), 483-7. https://doi.org/10.1016/j.jbmt.2013.03.006.

Wang, C., Bannuru, R., Ramel, J., Kupelnick, B., Scott, T., Schmid, C. H. (2010). Tai Chi on psychological well-being: systematic review and metaanalysis. BMC Complementary and Alternative Medicine 10(23), 1-16. https://doi. org/10.1186/1472-6882-10-23.

Wells, C., Kolt, G. S., Bialocerkowski, A. (2012). Defining Pilates exercise: a systematic review. Complement Ther Med. 20(4), 253-62. https://doi.org/10.1016/j.ctim.2012.02.005.

Yang, P. Y., Ho, K. H., Chen, H. C., Chien, M. Y. (2012). Exercise training improves sleep quality in middle-aged and older adults with sleep problems: a systematic review. Journal of physiotherapy, 58(3), 157-163. https://doi.org /10.1016/s1836-9553(12)70106-6. 